\title{
Atoms, quanta, and qubits: Atomism in quantum mechanics and information
}

\author{
Vasil Penchev, vasildinev@gmail.com \\ Bulgarian Academy of Sciences: Institute of Philosophy and Sociology: \\ Dept. of Logical Systems and Models
}

\begin{abstract}
The original conception of atomism suggests "atoms", which cannot be divided more into composing parts. However, the name "atom" in physics is reserved for entities, which can be divided into electrons, protons, neutrons and other "elementary particles", some of which are in turn compounded by other, "more elementary" ones. Instead of this, quantum mechanics is grounded on the actually indivisible quanta of action limited by the fundamental Planck constant. It resolves the problem of how both discrete and continuous (even smooth) to be described uniformly and invariantly in thus. Quantum mechanics can be interpreted in terms of quantum information. Qubit is the indivisible unit ("atom") of quantum information. The imagery of atomism in modern physics moves from atoms of matter (or energy) via "atoms" (quanta) of action to "atoms" (qubits) of quantum information. This is a conceptual shift in the cognition of reality to terms of information, choice, and time.
\end{abstract}

Key words: atom, quantum, qubit, quantum information, choice

\section{Motivation as introduction}

One can describe the conceptual shift in the philosophical reflection on the atomism in physics as the movement from the material atoms and the corresponding quantity of mass to the quantum bits (qubits) and corresponding quantity of (quantum) information via the fundamental conception and quantity of energy.

The contemporary physics shares two most fundamental theories: both general relativity and quantum mechanics, however each of the meeting the problem how to be consistent to the other one, or the cherished "quantum gravity" as the bridge between them or even their unification.

One may consider atomism as philosophically as historically in order to formulate ontologically the problem of quantum gravity in its terms:

The transition from discrete indivisible atoms having mass to quanta as discrete units of action according to the Planck constant as a conservative generalization implies a new auxiliary quantity to be introduced: its physical dimension is mas per time or "flow of mass" in a certain space point. If the flow of mass is granted, quantum mechanics needs at least two different values of it in each space point, i.e. the mapping of space to the field of that flow not to be any function in a classical sense.

On the contrary, general relativity manages to find a classical function to describe the same fact, however changing or "curving" the concept of space from Euclidean to pseudo-Riemannian one. Once this is so, one can generate isomorphism between the set of pairs of two different values of mass flow assigned to each space point, on the one hand, and the pairs of covariant and 
contravariant vector (definitively different for any nonzero curvature) associated to the same point after the "pseudo-Riemannian curving" of Euclidean space, on the other hand.

In other words, atomism directs to a certain class of solutions of the problem of quantum gravity: general relativity and quantum mechanics describe the same "state of affairs" in different ways, particularly involving rather different underlying mathematical structures, correspondingly, pseudo-Riemannian space and the separable complex Hilbert space.

The well-known precedent of that kind of solution is the unification of Heisenberg's matrix mechanics and Schrödinger's wave mechanics into the contemporary quantum mechanics.

\section{Prehistory and background: from Democritus and Leucippus to Dalton}

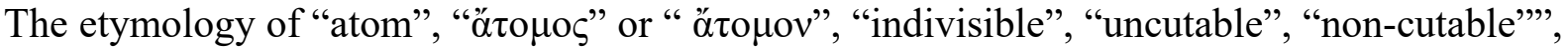
from $\dot{\alpha}$ - ("not") and $\tau \dot{\varepsilon} \mu \nu \omega$ ("I cut") is Ancient Greek. The original conception of atomism suggests that the process of dividing the matter has a limit: the "atoms", which cannot be more divided into composing parts. The matter itself is defined in turn as what consists of atoms.

The initial doctrine originates from Democritus and Leucippus, and perhaps earlier: “(a. Ps-GALEN History of Philosophy 3) A pupil of his [i.e., of Zeno's] was Leucippus of Abdera, the first proponent of the theory of atoms. Democritus got the theory from him and developed it further (Leucippus and Democritus [1999]: 54)".

A few main properties of Leucippus and Democritus' atom are:

1. There is nothing else than atoms and voids: "By convention sweet and by convention bitter, by convention hot, by convention cold, by convention color; but in reality atoms and void" (ibid. 9 [D16]).

2. Atoms are indivisible and unchangeable (ibid. 58).

3. The atoms distinguish from each other in form (ibid. 70)

4. Anything in the world is composed by atoms (ibid.).

5. All things differ from each other in the combination of atoms (ibid. 82).

In fact, Leucippus and Democritus' concept of atom is closer to the contemporary notion of atom in chemistry than in physics. John Dalton, a chemist, was who revived the ancient philosophical concept of atom for an experimental science such as chemistry. According to it:

1. The atom is the least unit of any chemical element defined by its mass.

2. The atoms cannot be decomposed, neither created.

3. They are identical: All chemical reactions are only some recombinations of atoms.

4. In other words, they are granted as the units of chemical matter.

Dalton developed its conception gradually: He introduced initially the (1802) "Table of the Weights of the different Gases constituting the Atmosphere" (Dalton 1805: 256), then the (1803) "Table of the relative weights of the ultimate particles of gaseous and other bodies" (Dalton 1805: 287) using yet the term "ultimate particles" for "atoms", and at last the term itself of atom (1808): "all bodies of sensible magnitude, whether liquid or solid, are constituted of a vast number of extremely small particles, or atoms of matter" (Dalton 1808: 141). 


\section{Atoms and motion in physics}

The concepts both of matter and of motion are fundamental for physics. However, their corresponding initial elements are quite different in the end of the 19 century: atoms and infinitesimals. The differentials of Leibniz and Newton rigorously reinterpreted in Robinson's nonstandard analysis (Robinson 1966: 2-4) can be thought as a kind of infinitely tiny atoms of physical motion. The discovery of quanta and the fundamental Planck constant in the beginning of the XX century forced different conceptions about the atomism and unity of matter and motion in physics.

Atomism and the concept of atom in physics began to diverge and mean different conceptions after the atom is divisible. The name "atom" in physics is reserved for entities, which can be decomposed into electrons, protons, neutrons and into some of the rest "elementary particles", some of which are in turn compounded by other, "still more elementary" ones. Furthermore, those ostensibly elementary particles turn out to be too much to be able to be really elementary: more than hundreds or even more than thousands.

As the matter should be single, the atoms of chemical matter were granted also as those of physical one.

\section{From atoms to elementary particles:}

However about a century later, the atoms were divided into parts. Even before that, the physical motion unlike any chemical reaction could not be ever represented as some recombinations of atoms. All atoms are discrete, but all physical motions are continuous and even smooth after Newton.

The Standard model is the last achievement of atomism in physics. After the Higgs boson has confirmed experimentally (The CMS Collaboration 2014: 557-558), the Standard model including 6 quarks, 6 leptons, and 13 bosons was ultimately corroborated. Just these, which can form all rest "elementary particles", are the fundamental and indivisible "atoms" both of physical matter and physical motion according to the Standard model.

The fermions in it (the quarks and leptons) might be interpreted as the "atoms of matter", and the bosons (the 8 gluons, photon, 2 W-boson, and Z-boson), as the "atoms of motion". The Higgs boson describes the relation between the two groups in a sense and thus, how mass at rest can arise.

\section{"Dark" and "entangled" clouds over the atomism of the Standard model}

However, the Standard model is not consistent enough to at least five rather well confirmed phenomena. Kelvin's metaphor about a few little clouds on the horizon of the classical physics (Kelvin 1901: 1-2) can be reinterpreted about the Standard model. These clouds are rather "dark" and "entangled", though:

1. Dark matter;

2. Dark energy;

3. Entanglement;

4. Gravity;

5. General relativity. 
A few details for each of them follow:

1. Dark matter:

Trimble demonstrated that the mass calculated by gravitational effects and the mass calculated by radiation differ considerably from each other (Trimble 1987: 451-452). An immense part of the mass in the universe should not emit any radiation therefore being namely dark matter. Only the neutrinos could be that dark matter according to the Standard model. The "Plank 2013 results" estimate the dark matter to be over 5 times more than the visible one (Planck collaboration and Tauber, 2013: 36, Table 9). The mass at rest of the neutrinos is almost zero. So, the ascription of more than $5 / 6$ of the mass of the universe to them is not convincing enough. The dark matter is not absolutely consistent to the Standard model, thus. If the Standard model does not describe all kinds of matter, the unknown ones would behavior as that dark matter.

Indeed gravity and thus the gravitational mass, which are described by general relativity, are beyond the Standard model. Therefore, they supply independent evidence, which is rather not in favor of the Standard model, though.

\section{Dark energy:}

"A current acceleration of the expansion" (Riess et al. 1998: 1009) of the universe is observed being "due not to a cosmological constant, but rather to a different, previously unknown physical entity that contributes to the universe's total energy density" and able to "lead to a different expansion history than the cosmological constant does" (Perlmutter et al. 1999: 584). This can be explained by dark energy, which should be about 69\% from the energy in the universe (Planck collaboration and Tauber 2013: 36, Table 9):

The rest belongs to the energetic equivalent of the "bright" and "dark" matter. Any energy, which is not any matter, cannot be dark according to the Standard model:

That energy should be visible for both gluons and photon are only stable bosons. Free gluons are almost impossible (except in "quark-gluon plasma") because of the phenomena of confinement, and the photons are "visible".

The dark energy is quite unexplainable in the framework of the Standard model. The dark matter is "only" not convincingly enough explainable in the framework of the Standard model. Unlike it, the dark energy is absolutely contradictory to it: no any particle with zero mass at rest, to which the phenomenon of "dark energy" might be due.

As dark matter as dark energy becomes "visible" only by astrophysical observations interpreted as usual in terms of general relativity.

\section{Entanglement:}

The entanglement was theoretically forecast in the famous paper of Einstein, Podolsky, and Rosen (Einstein et al. 1935: 777-780).

Schrödinger also forecast the phenomena of entanglement called by him "verschränkte Zustände" (Schrödinger 1935: 844-849).

Both papers deduced the phenomena of entanglement from the mathematical base of quantum mechanics, namely from the properties of Hilbert space. However, the former three demonstrated the forecast phenomenon as the proof of the alleged "incompleteness of quantum mechanics". 
The experimental confirmation of entanglement is precious and repeated many and many times, after

John Bell has deduced a sufficient condition as an experimentally verifiable criterion in order to distinguish classical from quantum correlation (Bell 1964: 195-200), or "entanglement". Aspect, Grangier, and Roger confirmed experimentally the existence of quantum correlations exceeding the upper limit of any possible classical correlations (Aspect et al. 1981: 460-463; Aspect et al. 1982: 91-94). All other experiments until now confirm categorically the phenomena of entanglement.

The conceptual bases of entanglement and the Standard model are rather inconsistent to each other. The Standard model is linear in principle as far as it is based on Hilbert space and its linear transformations. The Standard model is closed for it cannot be the linear part or approximation of some future non-linear generalization. Particularly, it cannot incorporate any additional symmetry as that would violate its linearity. The Standard model is ultimate knowledge in a sense and even thus necessarily incomplete.

\section{Gravity:}

Gravity is the only physical "force" (or interaction), which remains unexplained by any experimentally confirmed theory in the framework of the Standard model. Even more, any theory of gravity consistent with quantum mechanics, i.e. any theory of quantum gravity consistent with all relevant experiments does not exist. However, the hypothesis that quantum gravity cannot exist even as a law of nature would mean that quantum theory and quantum mechanics particularly are not universal doctrines about nature.

The attempts for gravity to be incorporated by the Standard model are unsuccessful until now. Gravity is a physical interaction, obviously, but it is not a part of the Standard model. Even more, it seems rather difficult to be any linear part of the Standard model because gravity is not local and thus linear in the sense of the Standard model. Even still more, the Standard model does not allow of any room in it at where gravity would stay: The Standard model is already closed: "No rooms!" That "closedness" originates immediately from its linearity and thus from the fundamental locality of all gauge theories. However, some elements of the Standard model (e.g. the neutrinos) might include implicitly gravity anyway.

\section{General relativity:}

Nevertheless, both general relativity and the Standard model are very well confirmed experimentally. General relativity might be called the "Standard model of gravity". Unfortunately, it seems to be not only non-quantum in principle but even not allowing of any consistent quantum generalization of it. However, it might be anyway incorporated as a breaking of symmetries in simile to the Higgs mechanism (Higgs 1964: 508-509). Furthermore, it supplies the interpretational framework, in which all existing experimental results against the Standard model such as the dark matter and energy can survive.

General relativity is the only "unoccupied" bastion of theoretical resistance against quantum mechanics. One can suggest that if the Standard model manages to incorporate it and entanglement, the problems with dark matter and energy will be resolved. 
The general relativity is at least quite different conceptually from the Standard model. Even general relativity and quantum mechanics underlying the Standard model are rather inconsistent to each other even as fundamental principles, on which they are built:

Quantum mechanics is linear, gaugeable and spatially local; quantumly invariant.

General relativity is just the opposite: nonlinear, non-gaugeable, spatially nonlocal; quantumly non-invariant.

\section{A few preliminary conclusions about atomism in physics}

They can be noticed concerning the following features:

I. The divisibility of the physical "atom";

II. The relation of the concepts of atoms and elementary particles;

III. The indivisibility of the Plank constant;

IV. The fundamental problem of quantum mechanics;

V. The probabilistic interpretation of quantum mechanics.

I. The divisibility of the physical "atom":

There is no physical principle, which can underlie the alleged indivisibility or "atomicity" of the atom itself. The "atom" can be considered as elementary only in relation to all chemical properties, but not at all as to the physical ones such as mass, energy, charge, momentum, trajectory, and other. The ostensibly indivisible atom turns out to be very complex composite system consisting of other "elementary particles" such as protons, neutrons, electrons, gluons, quarks and still more other.

\section{Atoms and elementary particles:}

The atoms and all elementary particles are pieces of mass or energy, which has to be conserved in closed systems according to the law of its conservation, but there is not any limit of its divisibility. The "revolution in physics" made by quantum mechanics introduced the Plank constant as the fundamental limit of divisibility of one physical quantity, action. Thus it managed to discover something, which is indivisible according to the laws of nature and even guaranteed by a fundamental constant, the Plank one.

Still more, physics and science have not managed to find until now nothing else also as fundamentally indivisible as the quantum of action.

III. The indivisibility of the Plank constant:

Quantum mechanics is grounded on the actually indivisible quanta of action limited by the fundamental Planck constant. Any other indivisibility in physics originates from the quantum of action. The meaning of that quant is so grandiose for knowledge of mankind that "quantum" is a synonym of "modern" or "contemporary" and antonym of "classical": e.g. quantum versus classical physics and even quantum versus classical science.

IV. The fundamental problem of quantum mechanics can be generalized as follows. Quantum mechanics resolves the problem of how both discrete and continuous (even smooth) to be described uniformly and invariantly. The quantum of action is what forced the physicists to generalize the description in the above way particularly to unify the quantum leaps and the smooth motions of classical physics including those studied by special and general relativity. 
$V$. The probabilistic interpretation of quantum mechanics reveals a rather unexpected and even unwelcome "bonus" originating from the resolving the above problem. Max Born demonstrated that the solution unifying discrete and smooth motion at the same time unifies the description of probable and actual states of quantum systems (Born 1926: 354) according to the sense of the socalled probabilistic interpretation of quantum mechanics. The fermions (having semi-integral spin) can be referred to the actual and therefore well-ordered state. The bosons (having integer spin) should describe the possible and unorderable coherent state:

\section{Quantum information and entanglement}

The key for the uniform description both of the discrete and continuous and of the possible and actual is Hilbert space underlying the contemporary formulation of quantum mechanics. So, the mathematical formalism based on Hilbert space is apt to satisfy the following conditions partly enumerated above:

a) A general description of all motions, either smooth or continuous, or discrete, as well as of the discrete of arithmetic and of the continuous of geometry at all;

b) A general description of any process in time, either in the future or in the present, or in the past as well as of the possible, actual, and necessary;

c) A general description of matter and motion and thus of any item in the universe.

The surprising bonuses, which Hilbert space supplies, continue, though. Hilbert space can be thought as a special kind of computer and thus all physical processes in the universe, as computations in this computer. In particular, Hilbert space can be represented as a quantum Turing tape. In fact, Hilbert space can be defined at least in a few different ways, one of which is that of quantum information as a "tape" of qubits instead of bits and processed by a quantum computer (quantum Turing machine) instead of a usual computer (standard Turing machine). Thus Hilbert space furthermore allows of any physical process to be interpreted as computational or informational, and the universe itself, as an omnipresent quantum computer.

There exists not only an analogy but a generalization from a classical computer to a quantum one and particularly from the "atom" of a usual computation, a bit, to a quantum bit, qubit. As a bit is the unit of classical information, as a qubit is the unit of quantum information.

'Qubit' is: $\alpha|0\rangle+\beta|1\rangle$ where $\alpha, \beta$ are two complex numbers such that $|\alpha|^{2}+|\beta|^{2}=1$, and $|0\rangle,|1\rangle$ are any two orthonormal vectors (e.g. the orthonormal bases of any two subspaces) in any vector space (e.g. Hilbert space, Euclidean space, etc.).

Both bit and qubit share some choice, which is between two alternatives for the former and among an infinite set for the latter.

Furthermore, the qubit can be discussed as the infinite generalization of a bit. The qubit can be realized as a kind of generalization of a bit as to infinite sets of alternatives in a choice instead of the two equally probable alternatives in a choice determining a bit of information. Consequently, the quantity of quantum information measured in units of qubits can be interpreted as the generalization of the quantity of classical information measured in units of bits. Thus, the quantity of information, whether classical or quantum, is the quantity of choices. 
The qubit is the natural atom of the universe. Both bit and qubit are indivisible units. Consequently, they should be considered as "atoms". Therefor they can be called "atoms" of information, whether classical or quantum. The indivisibility or "atomicity" of choice understood philosophically or mathematically underlies both: The choice can be well understood as an atom of time for the choice is the only which can transform future into past by means of the choice of the present. So the choice is an atom of time.

Wave function being a point in Hilbert space can be represented as a series of qubits. Any wave function or any state of a physical system can be seen as a corresponding sequence of those indivisible qubits or "atoms" of quantum information.

Indeed any physical system should be quantum according to quantum mechanics. Hilbert space can be also considered as the free variable of quantum information. Then any wave function is a value of that variable as what the Hilbert space has been considered.

Furthermore, the entanglement can be interpreted as a form of an immediate interaction of two or more wave functions. However, the entanglement cannot violate the indivisibility of the "atoms" of quantum information, the qubits constituting any wave functions.

Entanglement is defined as a state of a composite quantum system, when its corresponding Hilbert space cannot be factorized as any tensor product of the Hilbert spaces of the subsystems. Nevertheless, the entanglement does not shatter the qubits into any "parts".

The comparison of atoms, quanta, and qubits demonstrate the imagery of atomism in modern physics: from atoms of matter (or energy) via "atoms" (quanta) of action to "atoms" (qubits) of quantum information). That series of images of "atoms" can be understood as the direction of a generalization from the atoms of matter by itself to quanta shared both by the "particles of matter" (the fermions in the Standard model) to the "particles of motion and interaction" (i.e. the bosons there). Consequently, the concept of quanta is more fundamental than that of atoms.

Atomism is a framework or "reference frame" for the cognition of reality. The transformation from atoms to quanta is a conceptual shift in the cognition of reality. Reality cannot be understood more as some base of matter, which is in continuous motion. The "atoms" cannot refer only to that base of matter, neither the continuity only to the motion of matter. Time is what unifies matter and motion by the choice as a special kind of "temporal atom". The cognition of reality is gradually redirected to quanta, information, choice, and time in final analysis.

\section{The Standard model and the "Big Bang" in terms of quantum information}

The Standard model in turn can be reinterpreted in terms of quantum information. The number of particles (about fifty with the antiparticles) though exactly limited seems to be too big to be willingly accepted for "kinds of atoms". They call for some underlying and unifying principle. The so-called traditionally "elementary particles" are rather mathematical than physical entities. Thus, their nature should be rather informational than only physical or even only mathematical.

There is an asymmetry between the four physical interactions in relation to their origin and thus to their ability to be quantized: Two of them, namely the gravitational and electromagnetic one are a legacy from the continuous classical physics. The electromagnetic interaction is very successfully quantized unlike the gravitational one. The others, the weak and strong interaction, are initially 
quantum and do not need any quantization. Thus the Standard model includes them as well the quantized electromagnetic interaction excluding the annoying exception of gravity. One can see and test the reverse approach: both successfully quantized electromagnetic interaction and initially quantum weak and strong interaction to be represented in terms of the smooth motion of classical physics. Then the Standard model can be seen the quantum description of a privileged reference frame. It can be associated with just one privileged reference frame, which should be inertial for both Standard model and quantum mechanics are necessarily linear.

That privileged reference frame is identifiable as that of the "Big Bang". One can use the metaphor of the following "equation": It would equate the nonlinearity of general relativity to the linearity of Hilbert space. That "equation" would have the singularity of the beginning, i.e. the "Bing Bang" as the only solution.

The attitude of the theory of relativity to a privileged position is ambiguous. Special relativity arose in fighting against the "absolute" reference frame of the alleged "ether" and thus against any privileged reference frame. However, Einstein himself declared that general relativity unlike special one is consistent both to the availability and lack of some privileged reference frame(s) (Einstein 1920: 13-14). So, the "dethroned" ether can be restored as to general relativity according to him. Thus, the Standard model can be seen as the quantum doctrine about the restored ether and reference frame linked to it.

The privileged reference frame of the "Big Bang" is not necessary to be identified as that, which the Standard model needs. The Standard model needs only a privileged reference frame, which can be only inertial because of its linearity. However the Bing Bang requires furthermore still another condition ostensibly self-obvious. All physical laws and thus including the Standard model should be invariant to any reference frame. This implies the beginning (i.e. the Big Bang) as the only possible general reference frame as the only one common to all in the universe.

The privileged reference frame necessary for the Standard model allows of other interpretations, too. If the Standard model might be not so general and valid to any reference frame, other options appear. For example, one can suggest that inertial reference frame linkable to the present position of the earth to the universe as that privileged by the Standard model. Then all different enough reference frames in the universe would generate a proper Standard model relevant to each corresponding position to the universe.

The privileged inertial reference frame is mathematically isomorphic and thus equivalent in information to a single qubit. Indeed the information of a single qubit is exactly equal to that featuring an inertial reference frame. Even more, both are mathematically isomorphic to each other offering still evidence about the direct transition from informational to physical concepts.

One can see in detail how by means of few conceptual "equations":

$$
\text { A qubit }=\text { an inertial reference frame }
$$

A point of the ball $=$ the initial position of the reference frame A point on the surface (sphere) $=$ the velocity of the reference frame

The norm of the superposition, " 1 " = the light speed, " $c$ " 
The "multiverse", i.e. a plurality of parallel universes, can be placed in a single universe using both general relativity and many-words interpretation of quantum mechanics. Indeed the "Bing Bang" is one solution of the above "equation" however only under the additional condition of that the Standard model is universal at least for our universe. If one unifies the many-worlds interpretation of quantum mechanics (Everett III 1957: 454-462) with the smooth general relativity, the multiverse should be equivalent to the universe just as quantum mechanics unifies the discrete and smooth motion. Furthermore, any different enough reference frame in the universe should possesses a proper and different standard model. The multiverse implies a multi-model.

The qubit in turn can be decomposed into a few still more elementary choices. The qubit has been represented here until now as an atom of choice as an indivisible unit of quantum information. Nevertheless, it has a complex structure consisting of three independent choices, each of which being equivalent to the choice of an arbitrary real number. This is immediate corollary from the definition of "qubit": These three choices can be titled correspondingly and conventionally: first, second, and third.

Furthermore the three privileged "sub-choices" can be interpreted in terms of the Standard model. Each of those three choices can be seen as the source correspondingly of the "first, second, and third generation" of the fermions (i.e. the alleged particles of matter) of the Standard model. The strong interaction can be considered as the symmetry of the three single choices; the weak one, as the symmetry of the pairs of these three choices or as the symmetry of a 3-sphere; the electromagnetic one, by means of the single triple of these choices. As an example, the informational meaning of the electromagnetic interaction can be deduced as follows:

The sense of the Schrödinger equation can be interpreted in terms of quantum information so: Any transfinite bit is equal to exactly one quantum bit (qubit). That bit as an elementary choice is both indivisible unity of the two binary digits ("0" and " 1 ") and these digits separatedly. The magnetic component corresponds to the transformation of the former unity into a qubit, and the electric one, to the latter separation into the same qubit.

\section{Conclusions:}

1. The ancient atomism is revived in physics as the doctrine on quanta and quantum mechanics. The comparison of the concept of "atom" and "quantum" demonstrates that the "quantum" unifies matter and motion while the "atom" should be referred rather to matter only.

2. The Standard model is quantum: its description unifies matter (as fermions) and motion (as bosons). The Higgs boson determines the relation between them.

3. Quantum information allows of understanding the Standard model by means of the symmetries of exactly three underlying choices. These three choices constitute a privileged qubit equivalent to a privileged inertial reference frame. Quantum information decomposes the quanta to choices.

4. Choice is the atom of the being in final analysis. Choice in turn originates from time and its course from the unorderable or coherent future to the well-ordered or unchangeable past by the choices of the present. 


\section{References:}

Planck Collaboration and Tauber, J., (2014), "Planck 2013 results. I. Overview of products and scientific results," Astronomy and Astrophysics 571 (November 2014): A1.

Aspect, A. et al. (1981) "Experimental Tests of Realistic Local Theories via Bell's Theorem," Physical Review Letters 47 (7), 460-463.

Aspect, A. et al. (1982) "Experimental Realization of Einstein-Podolsky-Rosen-Bohm Gedanken Experiment: A New Violation of Bell's Inequalities," Physical Review Letters 49 (2): 91-94.

Bell, J. (1964) "On the Einstein - Podolsky - Rosen paradox," Physics (New York) 1 (3): 195-200.

Born, M. (1926) "Physical aspects of quantum mechanics," Nature 119: 354-357.

Dalton, J. (1805) "Experimental Enquiry into the Proportion of the several Gases or Elastic Fluids, constituting the Atmosphere," Memoirs of the Literary and Philosophical Society of Manchester (Second Series) 1: 244-258.

Dalton, J. (1805) "On the Absorption of Gases by Water and other Liquids," Memoirs of the Literary and Philosophical Society of Manchester (Second Series) 1: 271-287.

Dalton, J. (1808) A New System of Chemical Philosophy (Part 1), Manchester, printed by S. Russell for R. Bickerstaff, Strand, London.

Einstein, A. (1920) Äther und Relativitätstheorie, Berlin, Springer.

Einstein, A. et al, (1935) "Can Quantum-Mechanical Description of Physical Reality Be Considered Complete?” Physical Review 47 (10), 777-780.

Everett III, H. (1957) "Relative state" Formulation of Quantum Mechanics," Reviews of Modern Physics 29 (3): 454-462.

Higgs, P. (1964) "Broken symmetries and the masses of gauge bosons," Physical Review Letters 13 (16): 508-509.

Kelvin (1901) "Nineteenth-Century Clouds over the Dynamical Theory of Heat and Light," Philosophical Magazine (Series 6) 2 (7): 1-40.

Leucippus and Democritus (1999) The atomists, Leucippus and Democritus: fragments: a text and translation with a commentary (ed. Taylor, C.), Toronto, University of Toronto Press.

Peebles, P. and Ratra, B. (2003) "The cosmological constant and dark energy," Reviews of Modern Physics 75 (2): 559-606.

Perlmutter, S. et al. . (The Supernova Cosmology Project, 1999) "Measurements of Omega and Lambda from 42 high redshift supernovae," Astrophysical Journal 517 (2): 565-86.

Riess, A. et al. (Supernova Search Team, 1998) "Observational evidence from supernovae for an accelerating universe and a cosmological constant," Astronomical Journal 116 (3): 1009-38.

Robinson, A. (1966) Non-standard analysis, Amsterdam, North-Holland.

Schrödinger, E, (1935) “Die gegenwärtige situation in der Quantenmechanik," Die Naturwissenschaften, 23 (48): 807-812, 23 (49): 823-828, 23 (50): 844-849.

The CSM Collaboration (2014) "Evidence for the direct decay of the $125 \mathrm{GeV}$ Higgs boson to fermions," Nature Physics 10: 557-560.

Trimble, V. (1987) "Existence and nature of dark matter in the universe," Annual Review of Astronomy and Astrophysics 25: 425-472. 